\title{
Health-related quality of life and physical activity in persons at high risk for type 2 diabetes
}

\author{
ARJA HÄKKINEN ${ }^{1,2}$, ANNA KUKKA ${ }^{3}$, TANJA ONATSU ${ }^{2}$, SALME JÄRVENPÄ̈̈ ${ }^{4}$, \\ ARI HEINONEN ${ }^{2}$, HEIKKI KYRÖLÄINEN ${ }^{5}$, PABLO TOMAS-CARUS ${ }^{6} \&$ \\ MAURI KALLINEN ${ }^{7,8}$
}

${ }^{1}$ Department of Physical Medicine and Rehabilitation, Central Finland Health Care District, Fyväskylä, Finland, ${ }^{2}$ Department of Health Sciences, University of Fyväskylä, Fyväskylä, Finland, ${ }^{3}$ Finnish Physiotherapist Society, Helsinki, Finland, ${ }^{4}$ Medcare Foundation, Ä̈̈nekoski, Finland, ${ }^{5}$ Department of Biology of Physical Activity, University of Fyväskylä, Fyväskylä, Finland, ${ }^{6}$ Department of Sport and Health, University of Évora, Évora, Portugal, ${ }^{7}$ Central Finland Health Care District, Department of Rehabilitation, Fyväskylä, Finland, ${ }^{8}$ GeroCenter Foundation for Research and Development, Muurame, Finland

\begin{abstract}
Purpose. The aim of this study was to compare the health-related quality of life (HRQOL) of persons at risk for type 2 diabetes to that of the Finnish general population. In addition, the associations between physical activity and HRQOL at-risk persons were studied.

Methods. One hundred thirty-two at-risk persons were recruited from health care centres in Central Finland. Participants filled out questionnaires including demographic characteristics, HRQOL (SF-36), frequency of vigorous physical activity ( $\leq 2$ times a week, once a week, less than once a week) and comorbidities.

Results. HRQOL of at-risk persons differed significantly from that of the Finnish population in four of the eight dimensions of SF-36. Compared with reference values of the general population, the values of at-risk persons were worse for general health and pain, but better for mental health and role limitation (emotional) dimensions. Among at-risk persons those physically more active had less depressiveness and lower body weight. HRQOL decreased linearly with decreasing physical activity in all dimensions.

Conclusions. People with elevated risk for type 2 DM have reduced HRQOL in general health and body pain dimensions, but mental health and emotional role dimensions were better compared with that of the population. Among at-risk persons, the benefits of physical activity on HRQOL were seen in all HRQOL dimensions. Regular exercise and body weight control may improve subjective health and reduce risk for type $2 \mathrm{DM}$ and its consequences.
\end{abstract}

Keywords: Health-related quality of life, type II diabetes, physical activity, SF-36

\section{Introduction}

Type 2 diabetes is a metabolic disorder that is primarily characterised by insulin resistance, relative insulin deficiency and hyperglycemia. In 2003, the number of type $2 \mathrm{DM}$ patients in Finland was $1,87,000$. In addition it was estimated that the number of undiagnosed patients was $50,000-1,00,000$. It is rapidly increasing and assuming lifestyle remains unchanged, it is estimated that their number will rise up by $70 \%$ by the year 2010 . Type $2 \mathrm{DM}$ results from the interaction between genetic predisposition and behavioural and environmental risk factors [1]. Although the genetic basis of type 2 diabetes has yet to be identified, there is strong evidence that such modifiable risk factors as obesity and physical inactivity are the main general environmental determinants of the disease [2-4]. The more risk factors an individual has, the greater his/her likelihood of developing type 2 diabetes (see Appendix 1).

Correspondence: Arja Häkkinen, Department of Health Sciences, University of Jyväskylä, P.O. Box 35, 400145 Jyväskylä, Finland. Tel: +358-50-43-48-705. Fax: +358-14-260-2011. E-mail: arja.hakkinen@ksshp.fi

ISSN 0963-8288 print/ISSN 1464-5165 online $\odot 2008$ Informa UK Ltd.

DOI: $10.1080 / 08916930802354930$ 